\title{
Regulation of Energy Metabolism by Bone-Derived Hormones
}

\author{
Paula Mera, ${ }^{1}$ Mathieu Ferron, ${ }^{2}$ and Ioanna Mosialou ${ }^{1}$ \\ ${ }^{1}$ Columbia University Medical Center, New York, New York 10032 \\ ${ }^{2}$ Institut de Recherches Cliniques de Montréal, Montréal, Quebec H2W 1R7, Canada \\ Correspondence: mathieu.ferron@ircm.qc.ca
}

Like many other organs, bone can act as an endocrine organ through the secretion of bonespecific hormones or "osteokines." At least two osteokines are implicated in the control of glucose and energy metabolism: osteocalcin (OCN) and lipocalin-2 (LCN2). OCN stimulates the production and secretion of insulin by the pancreatic $\beta$-cells, but also favors adaptation to exercise by stimulating glucose and fatty acid (FA) utilization by the muscle. Both of these OCN functions are mediated by the G-protein-coupled receptor GPRC6A. In contrast, LCN2 influences energy metabolism by activating appetite-suppressing signaling in the brain. This action of LCN2 occurs through its binding to the melanocortin 4 receptor (MC4R) in the paraventricular nucleus of the hypothalamus (PVN) and ventromedial neurons of the hypothalamus.

\begin{abstract}
$A s$ a tissue unique to vertebrate, bone fulfills a Anumber of essential physiological functions. It acts as a scaffold for muscle and the other organs and hence allows locomotion. Bone is also an important reservoir for several minerals including calcium, phosphate, and magnesium (see Murshed 2017; Whyte 2017). Finally, bone cells are involved in the maintenance of the hematopoietic stem cell niche and, hence, in the production of lymphocytes, erythrocytes, and platelets (see Frenette 2017; Galán-Díez et al. 2017; Roodman 2017). All these functions of bones are regulated by specific hormonal and neuronal pathways acting on bone cells, which have been extensively covered in the literature.

Bone remodeling, the biological mechanism by which bone can grow, maintain its density, and repair fractures, involves the coordinated
\end{abstract}

bone-resorbing action of osteoclasts and the bone-forming action of osteoblasts (see Khosla and Monroe 2017; Kronenberg 2017). The nature of the cell biological processes involved strongly suggest that bone remodeling is an energy-consuming function and is therefore dependent on the energetic status of the whole organism. That bone remodeling is tightly linked to energy metabolism is best illustrated by the observation that anorexia nervosa, a state of low-caloric intake, is associated with osteoporosis in humans (Legroux-Gerot et al. 2005; Misra and Klibanski 2011). These studies illustrate a powerful relationship between energy intake and bone mass and suggest that a tight regulation of energy metabolism is required for optimal bone health. In line with these data, it was found that several hormones and neuropep-

Editors: Gerard Karsenty and David T. Scadden

Additional Perspectives on Bone: A Regulator of Physiology available at www.perspectivesinmedicine.org

Copyright (C) 2018 Cold Spring Harbor Laboratory Press; all rights reserved; doi: 10.1101/cshperspect.a031666 Cite this article as Cold Spring Harb Perspect Med 2018;8:a031666 
P. Mera et al.

tides implicated in the control of energy metabolism, first leptin but also insulin, adiponectin, NPY, orexins, and AGRP, also influence bone remodeling (Ducy et al. 2000; Cornish et al. 2002; Fulzele et al. 2010; Kajimura et al. 2013; Wei et al. 2014b; Kim et al. 2015; Horsnell and Baldock 2016). That bone is a metabolically active tissue whose functions are influenced by hormones involved in the control of energy metabolism together with the general principle of endocrine feedback loops, led researchers to postulate that bone itself may possess some endocrine functions influencing energy metabolism. This hypothesis was verified when it was shown that osteocalcin (OCN) is a bone-derived hormone influencing insulin secretion, insulin sensitivity, and muscle function during exercise. More recently, lipocalin-2 (LCN2) was identified as a second bone-specific hormone involved in the control of appetite and glucose metabolism. Hence, bone is a bona fide endocrine organ that influences energy metabolism through the production of at least two hormones. This article will review in detail the mechanisms of action and the regulation of OCN and LCN2.

\section{OSTEOCALCIN IS A BONE-DERIVED HORMONE REGULATING GLUCOSE METABOLISM}

OCN is a short protein, 46 amino acids long in the mouse and 49 amino acids long in humans, produced and secreted specifically by osteoblasts, the bone-forming cells. Inactivation of the two genes encoding OCN (Bglap1 and Bglap2) in mice has profound metabolic consequences (Lee et al. 2007). Most strikingly, OCNdeficient mice $\left(\mathrm{OCn}^{-1-}\right)$ are characterized by an increased adiposity, reduced peripheral insulin sensitivity, and decreased glucose tolerance. They also display liver steatosis and signs of inflammation in the liver and in white adipose tissue. Finally, the absence of OCN is associated with a reduction in global energy expenditure, which most likely contributes to the increased fat mass of these animals. Intermittent injections of recombinant OCN in lean or obese mice or rats resulted in phenotypes opposite to the ones observed in the $\mathrm{Ocn}^{-l-}$ mice, that is, they increased energy expenditure, reduced fat mass, improved insulin sensitivity, and prevented liver steatosis (Ferron et al. 2008, 2012; Gupte et al. 2014; Huang et al. 2017). Similarly, the mouse models in which the circulating level of the active form of OCN is increased, for example, $E s p^{-/-}$or osteoblast-specific knockout of $G g c x$ (see also sections on endocrine function and bone resorption) (Fig. 2), are also characterized by improved insulin sensitivity, reduced fat accumulation, and increased energy expenditure (Lee et al. 2007; Ferron et al. 2015).

The mechanisms by which OCN promotes insulin sensitivity and energy expenditure in vivo are still not fully understood. OCN appears to promote directly in white adipocytes the expression of adiponectin, a hormone that may favor peripheral insulin sensitivity in obese animals (Yamauchi et al. 2001). However, adiponectin does not function as an insulin-sensitizing hormone in lean mice (Kajimura et al. 2013). In addition, OCN suppresses lipolysis, promotes directly glucose uptake, and can suppress the secretion of proinflammatory cytokines in white adipocytes (Fig. 1) (Lee et al. 2007; Hill et al. 2014). OCN also promotes mitochondria biogenesis in muscles when injected in obese mice (Ferron et al. 2012) and stimulates the expression of genes involved in thermogenesis $(P g c 1 \alpha$ and Ucp1) in brown adipocytes in vivo and ex vivo (Ferron et al. 2008). Altogether, these observations suggest that the protective effect of this hormone on obesity and insulin resistance could be a result, in part, of its capacity to enhance energy expenditure in muscle and brown adipose tissue.

In addition to these various metabolic anomalies, $\mathrm{Ocn}^{-1-}$ animals also displayed reduced circulating insulin levels in response to glucose (Lee et al. 2007). This decrease in insulin is explained by a dual effect of OCN on pancreatic $\beta$ cells, whereby it promotes insulin synthesis and secretion on the one hand and $\beta$-cell proliferation on the other hand (Ferron et al. 2012; Wei et al. 2014a). Treatment of primary mouse islets with recombinant $\mathrm{OCN}$ increases the expression of the cell-cycle regulators Ccnd1, Ccnd2, and Cdk4 (Ferron et al. 2008). Moreover, OCN directly enhances the transcription of the 
Regulation of Energy Metabolism by Bone-Derived Hormones
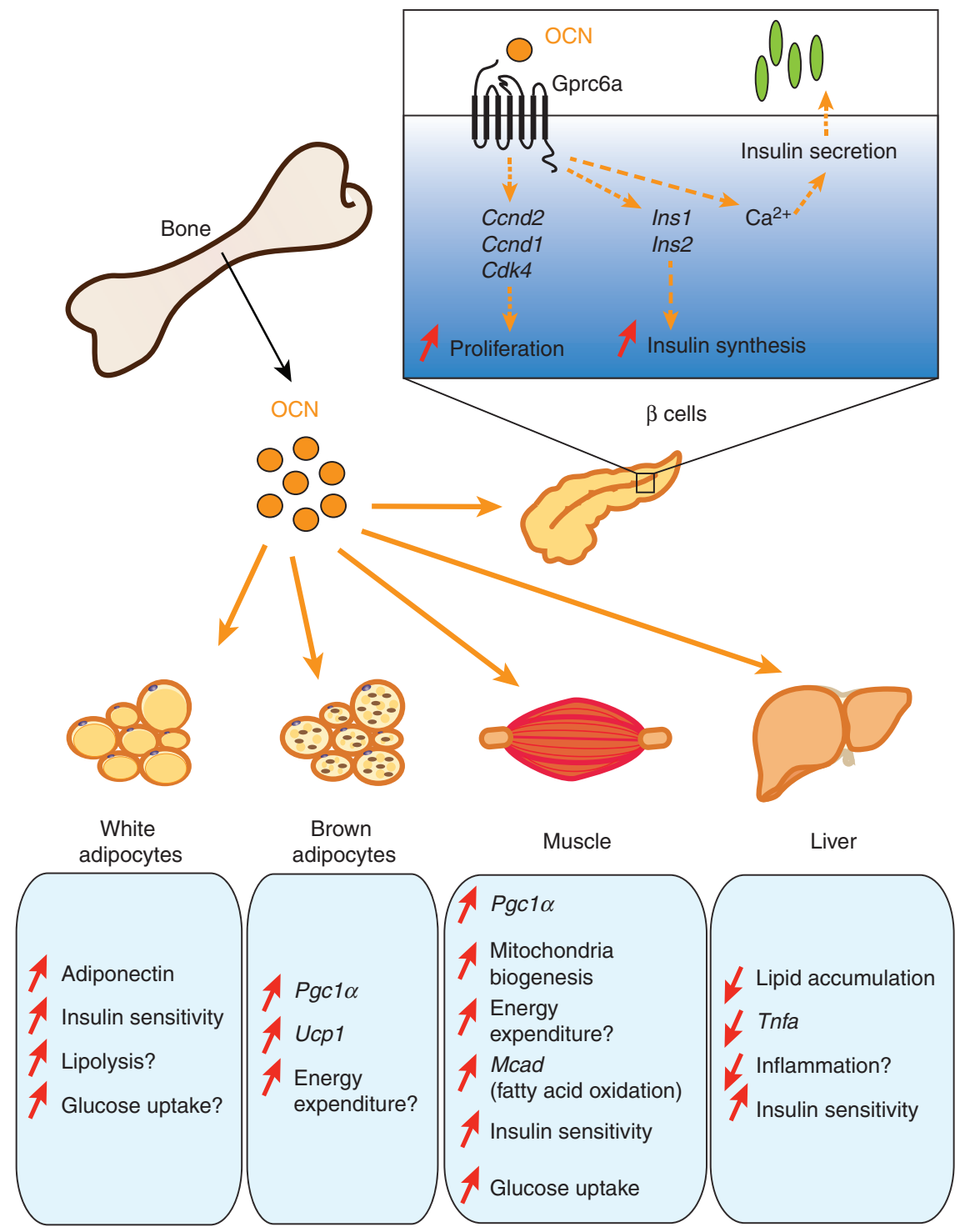

Figure 1. Endocrine functions of osteocalcin (OCN). Once released in the bloodstream, undercarboxylated bioactive OCN affects glucose metabolism mainly in two ways. First, OCN directly affects $\beta$-cell function by binding to the receptor GPRC6A and increasing their capacity to proliferate as well as to synthetize and secrete insulin. Second, OCN improves insulin sensitivity and energy expenditure through multiple mechanisms. OCN stimulates energy expenditure by increasing mitochondrial biogenesis in the muscle and by regulating the expression of genes implicated in energy consumption in brown adipose tissue and skeletal muscle. OCN also affects insulin sensitivity possibly by increasing adiponectin expression in white fat and decreasing lipid accumulation and inflammation in steatotic liver. A direct impact of $\mathrm{OCN}$ as an insulin-sensitizing hormone is speculative and remains to be established. 
P. Mera et al.

two mouse insulin genes, Ins1 and Ins2 (Ferron et al. 2008; Wei et al. 2014a). The acute effect of OCN on insulin secretion appears to be mediated by $\mathrm{Ca}^{2+}$ signaling in $\beta$ cells (Fig. 1) (Hinoi et al. 2008).

Several cell-based assays confirmed the notion gathered in vivo that OCN stimulates insulin secretion in rat and in human islets ex vivo (Kover et al. 2015; Sabek et al. 2015; Gao et al. 2016a,b). Genetic studies further support an impact of OCN polymorphisms in glucose and energy metabolism in humans (Das et al. 2010; Korostishevsky et al. 2012). Finally, two metaanalysis showed, respectively, that circulating levels of total OCN are higher in subjects with normal glucose tolerance compared with patients with type 2 diabetes (Kunutsor et al. 2015) and that OCN serum level was an independent risk factor for the development of type 2 diabetes (Liu et al. 2015).

\section{GPRC6A MEDIATES OSTEOCALCIN FUNCTIONS IN $\beta$ CELLS}

Like most known peptide hormones, OCN mediates its function through the binding of at least one specific receptor: GPRC6A (Fig. 1). This orphan G-protein-coupled receptor (GPCR) shares some sequence identity with the calcium-sensing receptors (CASRs), which are involved in calcium homeostasis through the regulation of parathyroid hormone $(\mathrm{PTH})$ release (see Kronenberg 2017). GPRC6A was first characterized as a cation-sensing receptor, and more recently as a putative receptor for amino acids, steroids, and OCN (Pi and Quarles 2012; Wei et al. 2014a). That Gprc6a $a^{-1-}$ mice phenocopied OCN-deficient animals with regard to their defects in insulin secretion and glucose tolerance indicate that GPRC6A is the receptor for OCN at least in pancreatic $\beta$ cells (Pi et al. 2008). Inactivation of Gprc6a specifically in the pancreas resulted in reduced $\beta$-cell proliferation and decreased insulin secretion in response to glucose (Wei et al. 2014a; Pi et al. 2016). That OCN capacity to induce insulin secretion is abrogated in $\mathrm{Gprc6a}^{-/-}$islets further showed that GPRC6A is the bona fide OCN receptor in $\beta$ cells.
GPRC6A also acts as an OCN receptor in Leydig cells, where OCN signaling promotes testosterone synthesis (Oury et al. 2011; De Toni et al. 2014). It should be noted that even if both the $\mathrm{Ocn}^{-/-}$and the $\mathrm{Gprc}^{-1-}$ mice are characterized by an increased fat mass, a receptor of $\mathrm{OCN}$ in adipocytes has not been identified yet.

Several lines of evidence suggest that the GPRC6A function as an OCN receptor is conserved in humans. First, human OCN can bind to and activate human GPRC6A receptor as it was reported for the mouse protein (De Toni et al. 2016b). Second, a mutation in the human GPRC6A gene affects trafficking to the plasma membrane and is associated with insulin resistance and testis failure in humans, two phenotypes also observed in OCN-deficient mice (Oury et al. 2013a). Third, polymorphisms in the human GPRC6A gene were shown to be also associated with insulin resistance and testis failure (De Toni et al. 2016a; Di Nisio et al. 2017).

\section{THE ENDOCRINE FUNCTION OF OSTEOCALCIN IS REGULATED BY $\gamma$-CARBOXYLATION}

Before it is secreted by the osteoblast, OCN is $\gamma$-carboxylated on three glutamic acid residues (Glu), which are thereby converted to $\gamma$-carboxyglutamic acid (Gla). This posttranslational modification occurs in the endoplasmic reticulum (ER) of the osteoblasts and is accomplished by the $\gamma$-glutamyl carboxylase (GGCX), an enzyme requiring reduced vitamin $\mathrm{K}$ as a cofactor (Fig. 2A). For each carboxylation reaction, one molecule of reduced vitamin $\mathrm{K}$ is oxidized to vitamin K epoxide by GGCX. A second enzyme called vitamin $\mathrm{K}$ epoxide reductase (VKORC1) is responsible for reducing the vitamin $\mathrm{K}$ epoxide, allowing the recycling of the vitamin $\mathrm{K}$ and further $\gamma$-carboxylation reactions. The presence of Gla residues in OCN allows its binding with high-affinity to hydroxyapatite the mineral component of bone, and probably explains why the majority of carboxylated OCN (Gla-Ocn) accumulates in the bone extracellular matrix where it is the most abundant noncollagenous 
A
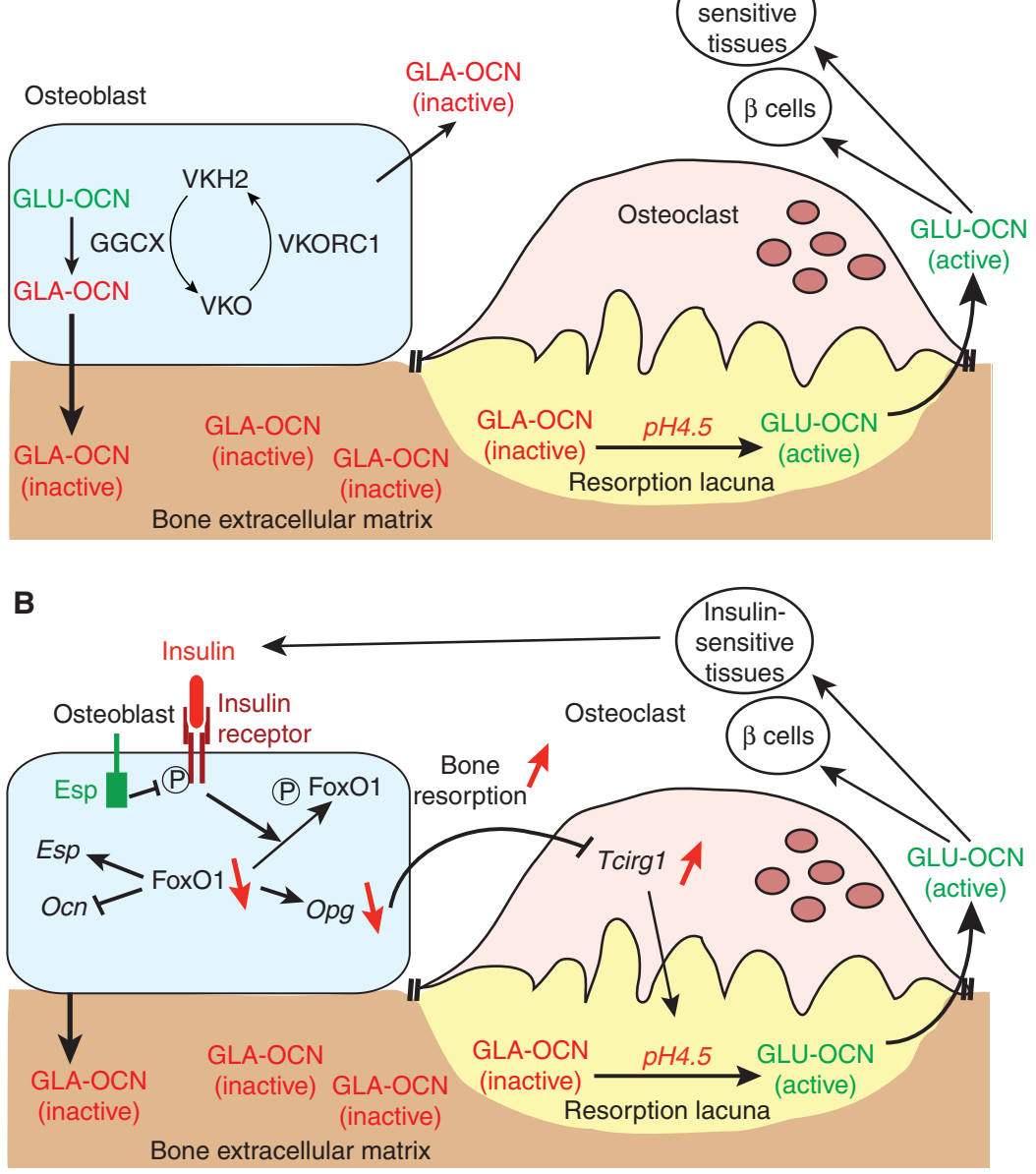

Figure 2. Regulation of osteocalcin (OCN) activity. (A) Once $\gamma$-carboxylated and secreted by osteoblasts, OCN is stored in the bone extracellular matrix in an inactive form. To fulfill its beneficial effects on glucose metabolism, OCN has to be activated, that is, decarboxylated. This is accomplished through osteoclastic bone resorption that generates the acid $\mathrm{pH}$ necessary for OCN decarboxylation. (B) Insulin signaling in osteoblasts affects OCN activity by increasing bone resorption through osteoprotegerin $(O p g)$ down-regulation. Because OCN stimulates insulin secretion, a feedforward loop exists between OCN and insulin activity. Esp, a tyrosine phosphatase, negatively regulates insulin receptor signaling and decreases OCN activity.

protein (Fig. 2A). In the circulation, OCN exists in two forms: carboxylated OCN (Gla-Ocn) and undercarboxylated (Glu13-Ocn or Glu-Ocn). In vitro and in vivo studies in mice have established that $\gamma$-carboxylation of OCN, particularly on the first Glu residue (Glu13 in mice and Glu17 in humans), inhibits OCN endocrine action on energy metabolism and that undercarboxylated OCN is the bioactive form of this hormone (Ferron et al. 2008; Zhou et al. 2013). For instance, inactivation of GGCX in osteo- blasts results in increased circulating levels of undercarboxylated $\mathrm{OCN}$ and improved glucose tolerance in mice (Ferron et al. 2015). A crosssectional study in postmenopausal obese women also showed that the level of OCN carboxylation on its Glu17 residue positively correlates with insulin resistance and low-grade inflammation (Bonneau et al. 2017), suggesting that $\gamma$-carboxylation of OCN negatively regulates the function of this hormone in humans as it does in rodents. 
P. Mera et al.

\section{OSTEOCALCIN IS DECARBOXYLATED AND ACTIVATED DURING BONE RESORPTION}

It turns out that the decarboxylation of $\mathrm{OCN}$ is a nonenzymatic process that occurs outside the osteoblasts during bone resorption by osteoclasts (Fig. 2B). It is the low pH ( 4.5) that is generated by the osteoclasts in the resorption lacuna that decarboxylates at least one of the Gla residues present in OCN (Ferron et al. 2010), thus allowing the release of bioactive OCN (Glu13-OCN). Accordingly, mice with increased bone resorption also display increased circulating levels of bioactive OCN and improved glucose tolerance and insulin sensitivity, whereas mice lacking osteoclasts have reduced levels of bioactive OCN and decreased glucose tolerance (Lacombe et al. 2013). Interestingly, bone resorption couples insulin and $\mathrm{OCN}$ in a feedforward loop controlling glucose metabolism. Indeed, insulin signaling in osteoblasts, through the inhibition of the transcription factor FOXO1, reduced the expression of Osteoprotegerin (OPG), a negative regulator of bone resorption (Ferron et al. 2010; Rached et al. 2010), thereby inducing increased osteoclastic activity and the release of bioactive OCN (Fig. 2B).

\section{A ROLE FOR OSTEOCALCIN IN ADAPTATION TO EXERCISE}

A question raised by the favorable influence of OCN on glucose homeostasis is whether there is any difference between $\mathrm{OCN}$ and insulin functions. Considering the significant role of skeletal muscle in the maintenance of normal wholebody glucose homeostasis, it became important to determine whether OCN influences any aspect of muscle energy metabolism. One experimental approach to address this question was to study the functions of any OCN during exercise, a physiological situation characterized by an increase in skeletal muscle glucose uptake and a concomitant decrease in circulating insulin levels. That circulating levels of bioactive OCN double after a single bout of endurance exercise in mice and in humans, was another reason to address this question. In the mouse, this increase in bioactive $\mathrm{OCN}$ is caused, in part, by an increase in bone resorption (Mera et al. 2016a).

\section{OSTEOCALCIN REGULATES SKELETAL MUSCLE ENERGY METABOLISM DURING EXERCISE}

A common feature to all known functions of $\mathrm{OCN}$ is the fact that they decline relatively early during adult life (Lee et al. 2007; Oury et al. 2011, 2013b). This observation was another reason to ask whether OCN regulates skeletal muscle function and adaptation to exercise, a physiological process that is severely affected early during aging. What made this hypothesis attractive is that circulating levels of bioactive OCN decrease early during aging in mice, monkeys, and humans of both genders and do not increase after exercise to the same extent in older mice as in young ones. Moreover, at least in mice, this decrease in circulating bioactive OCN occurs at the same time as the ability to perform exercise declines (Mera et al. 2016a). All these observations were an incentive to test whether the administration of exogenous OCN to wild-type (WT) mice might increase their ability to exercise. A single injection of exogenous OCN immediately before exercise or a chronic delivery of this hormone for 1 month not only increased the exercise capacity of young mice but also restored aerobic endurance in older mice to the level seen in young adult mice (Mera et al. 2016a). Moreover, chronic delivery of OCN also favors an increase in muscle mass in older mice (Mera et al. 2016b). These experiments highlighted the therapeutic potential of OCN to reverse the age-induced decline in exercise capacity and muscle mass observed in mice, but also in humans.

The identification of GPRC6A as a receptor for OCN in peripheral organs provided the necessary tool to study the role, if any, of OCN signaling in skeletal muscle aside from its functions in other organs. Analysis of 3-month-old $\mathrm{Ocn}^{-1-}$ mice and mice lacking GPRC6A only in myofibers ( $\mathrm{Gprc} 6 \mathrm{M}_{\mathrm{Mck}}{ }^{-1}$ ) revealed that when forced to run on a treadmill at a constant speed and until exhausted, $\mathrm{Ocn}^{-1-}$ and $\mathrm{Gprc} \mathrm{a}_{\mathrm{Mck}}{ }^{-1-}$ mice run $20 \%$ to $30 \%$ less than control litter- 
Regulation of Energy Metabolism by Bone-Derived Hormones

mates (Mera et al. 2016a). Further investigation of this phenotype reveals that OCN regulates the uptake and catabolism of glucose and fatty acids (FAs) in muscle during exercise (Fig. 3). In that respect, and this is a point of fundamental importance, OCN differs from insulin, which is an anabolic hormone. The uptake and utilization of these nutrients by contracting myofibers is absolutely essential for the ability to exercise. Therefore, the regulation of skeletal muscle nutrient catabolism by OCN certainly explains, at least in part, the decreased exercise performance observed in $\mathrm{Ocn}^{-1-}$ and $\mathrm{Gprc}^{-1} a_{\mathrm{Mck}}{ }^{-1-}$ mice.

OCN supports muscle function during exercise through an additional mechanism: it favors the expression and release of interleukin 6 (IL-6), the first molecule found to be secreted into blood in response to muscle contraction and one of the first myokines ever identified (Steensberg et al. 2000; Pedersen and Febbraio
2008). Initially, it was observed that its circulating levels increase after exercise in humans and rodents in a manner that is proportional to the length of exercise and the amount of muscles involved (Febbraio and Pedersen 2002; Febbraio et al. 2004; Nielsen et al. 2007). Additionally, it was shown that IL-6 is expressed in cultured myotubes and myofibers, and that skeletal muscle is the major source of circulating IL-6 during exercise (Keller et al. 2001; Steenbergen et al. 2002). During exercise, IL-6 acts in an autocrine, paracrine, and endocrine fashion to promote skeletal muscle nutrient utilization, glucose production in the liver, and lipolysis in the white adipose tissue (Pedersen and Febbraio 2008). Taking into consideration experimental observations showing that IL-6 also signals in bone cells (Tamura et al. 1993), the regulation of IL- 6 by OCN during exercise raised another fundamental question: could IL-6 signal back

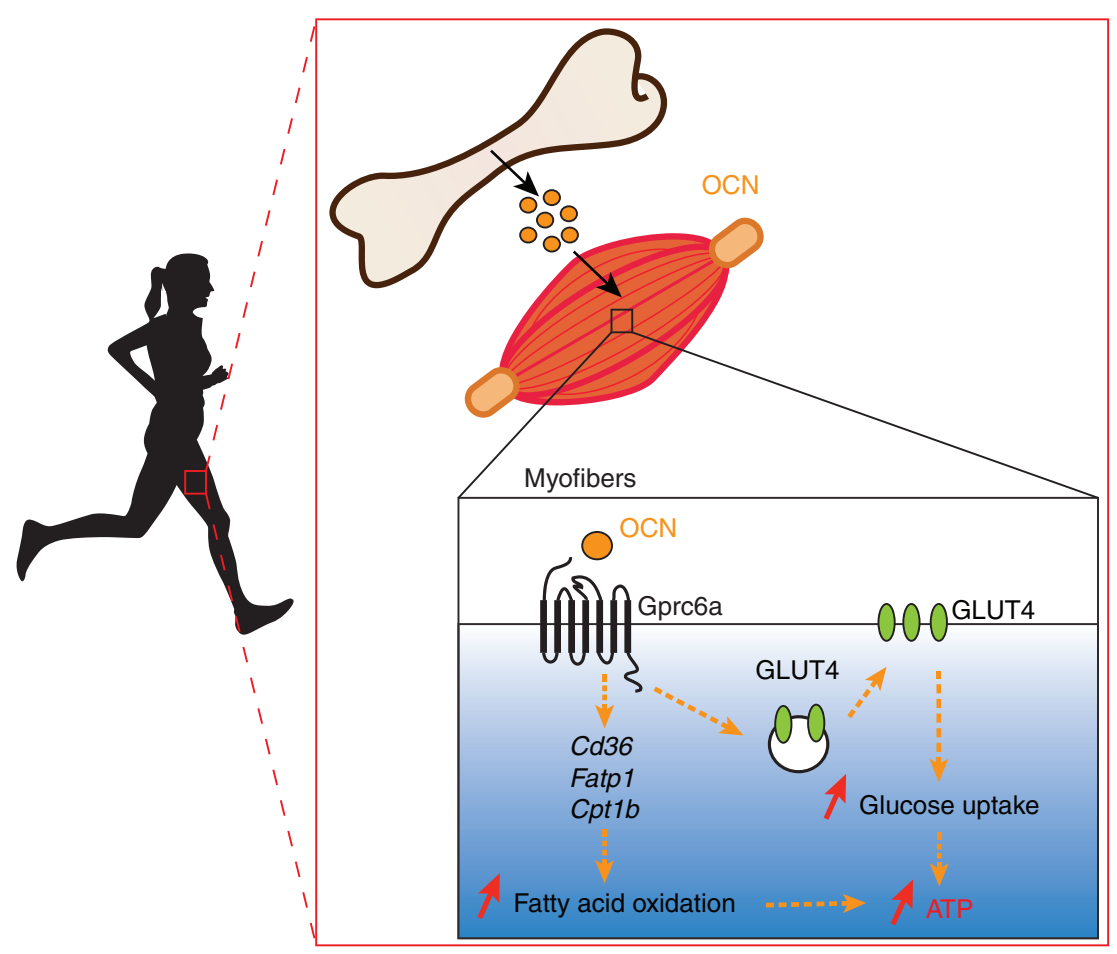

Figure 3. Mechanism of action of osteocalcin $(\mathrm{OCN})$ in skeletal muscle during exercise. During exercise, bioactive OCN is released by osteoblasts and binds to the receptor GPRC6A in myofibers where it promotes the uptake and utilization of nutrients. First, OCN favors the expression of fatty acid (FA) transporters and stimulates $\beta$ oxidation. Second, OCN favors the translocation of the glucose transporter GLUT4 to the plasma membrane. This, in turn, allows the increase in glucose uptake and catabolism. 
P. Mera et al.

to bone to modulate the production of bioactive $\mathrm{OCN}$ ?

\section{A CROSS TALK BETWEEN BONE AND SKELETAL MUSCLE MODULATES ADAPTATION TO EXERCISE}

Interestingly, blood markers of bone resorption and bioactive OCN increase in WT mice but not in IL-6-deficient mice $\left(\mathrm{Il6}^{-1-}\right)$ after a single bout of exercise (Mera et al. 2016a). These findings suggested that IL- 6 might act on bone cells to fulfill these functions. Cell-based experiments revealed that the addition of IL-6 increases the expression of Rankl, a cytokine that favors osteoclasts differentiation, and decreases Osteoprotegerin $(O p g)$, a decoy receptor for RANKL and an inhibitor of bone resorption (Teitelbaum and Ross 2003) in cultured osteoblasts (Mera et al. 2016a). These results suggest that in vivo IL-6 might act during exercise in cells of the osteoblasts lineage to increase bone resorption and the production of bioactive OCN. Extensive genetic and molecular studies are now required to fully understand the regulation of bioactive OCN by IL- 6 during exercise.

Altogether, the analyses of $\mathrm{Ocn}^{-1-}$, Gprc6 $a_{M c k}{ }^{-1-}$, and $\mathrm{Il6}^{-1-}$ mice revealed the existence of a feedforward loop between bone (via OCN) and muscle (via IL-6) that promotes adaptation to exercise through at least three different synergistic mechanisms (Fig. 4). First, OCN increases nutrient uptake and catabolism in myofibers. Second, OCN signaling in myofibers promotes the expression and secretion of IL-6; this, in turn, might allow the generation of extramuscular glucose and FAs (van Hall et al. 2003; Febbraio et al. 2004). Third, IL-6 increases the production of bioactive OCN. This model does not exclude the possibility that OCN and IL-6 might have additional functions to modulate adaptation to exercise. Likewise, other molecules made in bone cells or skeletal muscle cells could contribute to this cross talk.

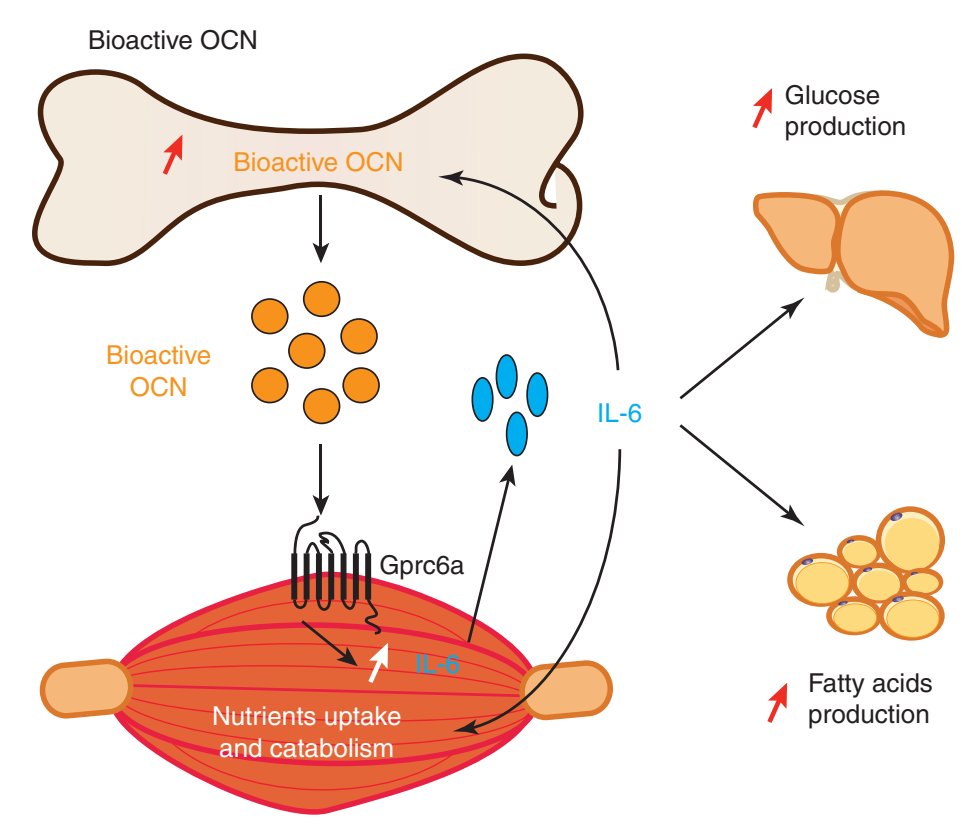

Figure 4. A cross talk between bone, via osteocalcin (OCN), and skeletal muscle, via interleukin 6 (IL-6), promotes adaptation to exercise. Circulating levels of bioactive OCN increases during exercise. OCN signals in myofibers through GPRC6A, in which it induces the expression of $I l 6$ and the increase in circulating levels of this myokine. IL-6 signals back to skeletal muscle where it favors glucose and fatty acid (FA) utilization. IL-6 also stimulates FA production in the white adipose tissue and glucose production in the liver. Furthermore, IL-6 signals to bone to increase the production of bioactive OCN. 
Regulation of Energy Metabolism by Bone-Derived Hormones

IL-6 and OCN both regulate similar aspects of skeletal muscle metabolism during exercise, for example, the increase in glucose and FA catabolism. Experiments studying the effect of exogenous OCN on glucose and FA utilization in WT and $I l 6^{-1-}$ cultured myofibers and myotubes showed that OCN can induce these functions in a similar manner in control and Il6-deficient cells (Mera et al. 2016a), showing that OCN is acting independently of IL- 6 to promote nutrient utilization in myofibers. Whether OCN and IL-6 might have synergistic function to increase glucose and FA uptake and utilization in skeletal muscle during exercise remains to be established.

\section{BONE AS A REGULATOR OF APPETITE: THE ANOREXIGENIC FUNCTION OF OSTEOBLAST-DERIVED LIPOCALIN-2}

The discovery of the endocrine functions of OCN established a new paradigm whereby proteins secreted from osteoblasts act on distal organs to influence whole-body energy metabolism. It also raised the question of whether additional bone-derived hormones may exist and contribute to the regulation of energy metabolism. To address this question, osteoblasts were conditionally ablated in mice. As expected, a 50\% decrease in osteoblast numbers decreased OCN levels and compromised glucose metabolism. However, and more intriguingly, an additional metabolic function was affected: appetite. Decreased osteoblast numbers increased food intake, a function that is not regulated by OCN (Yoshikawa et al. 2011). These findings identified appetite as another metabolic function regulated by osteoblasts.

The identification of the hormone responsible for this function was achieved by taking advantage of another mouse model of bone-related improved energy metabolism, mice lacking Foxo1 in osteoblasts (Rached et al. 2010). FOXO1 is a transcription factor regulating osteoblast function, and its effects on energy metabolism are the result, only in part, of its ability to regulate OCN activity. This raised the possibility that additional hormones involved in energy metabolism may be targets of FOXO1. A comparative gene expression analysis revealed that the expression of the gene encoding lipocalin-2 (LCN2) was increased in Foxo 1-deficient osteoblasts. Circulating levels of LCN2 were also increased in FoxO1-null mice. LCN2, also known as neutrophil gelatinase-associated lipocalin, is a secreted glycoprotein that was previously thought to be an adipokine (Yan et al. 2007). However, expression profiling showed that Lcn2 is expressed at least 10 -fold higher in bone than in fat or any other tissue. This observation led to the generation of mice lacking Lcn2 specifically in osteoblasts (Lcn2osb ${ }^{-1-}$ mice) to characterize its metabolic function (Mosialou et al. 2017). Analysis of these mutant mice showed that inactivation of Lcn 2 in osteoblasts increases by $16 \%$ food intake. As a consequence of this increase in food intake, Lcn $2 \mathrm{osb}^{-1-}$ mice show increased adiposity and body weight, lower glucose tolerance, and insulin sensitivity, and a decrease in circulating insulin levels. Islet number and size, $\beta$-cell mass and proliferation, and insulin secretion are also decreased in $L c n 2 \mathrm{osb}^{-1-}$ mice. Interestingly, the increase in food intake precedes changes in blood glucose levels and body weight and partly contributes to the development of impaired glucose metabolism because normalization of food intake by pair-feeding $L c n 2 \mathrm{osb}^{-1-}$ to WT littermates restores body weight, fat mass, and insulin sensitivity. However, this manipulation cannot fully rescue the decrease in serum insulin levels and insulin secretion following glucose load and, as a result, glucose intolerance persists. Cell culture experiments revealed that LCN2 acts directly on $\beta$ cells to favor their proliferation and insulin secretion. Global deletion of $L c n 2$ results in the same metabolic abnormalities, whereas mice with adipocyte-specific deletion of Lcn2, which contributes only a $30 \%$ decrease in circulating LCN2 levels, show normal food intake and body composition. These observations confirm that osteoblasts are the cells that contribute LCN2 levels sufficient to regulate appetite and glucose metabolism, at least in basal states.

In support of this contention, using a different mouse model of germline deletion of $L c n 2$, several studies reported a beneficial role for LCN2 in energy metabolism. For instance, it was shown that LCN2 protects from diet-in- 
P. Mera et al.

duced obesity, fatty liver disease, atherogenic dyslipidemia and insulin resistance, suppresses hepatic gluconeogenesis and promotes adaptive thermogenesis, activation of brown adipose tissue, and FA oxidation (Guo et al. 2010, 2012, 2013; Paton et al. 2013; Zhang et al. 2014). Improved FA oxidation has also been found in normal-weight women in whom LCN2 serum levels correlate with energy expenditure measured after a high-fat meal (Paton et al. 2013). In contrast, one study reported a minimal effect of $L c n 2$ deficiency in glucose homeostasis with no effects on body weight (Jun et al. 2011), whereas another showed a protection from aging and obesity-induced insulin resistance (Law et al. 2010). Differences in targeting strategy and mainly the retention of a PGK-neo cassette in the latter studies, which is shown to disrupt the expression of other genes located near the intended target (Olson et al. 1996), may account for differences in the phenotypes developed. Nevertheless, the fact that chronic administration of exogenous LCN2 in lean and obese mice decreases food intake, fat mass, and body weight gain, and improves glucose homeostasis and energy expenditure (Mosialou et al. 2017) provides further evidence that LCN2 regulates energy metabolism.

Suggesting a physiological role of LCN2 in the regulation of feeding, osteoblastic expression and circulating LCN2 levels increase threefold 1 to 3 hours postprandially in mice. This increase in serum levels correlates with a suppression of food intake. Lcn2osb ${ }^{-1-}$ mice had higher rebound food intake following fasting while restoration of LCN2 levels on refeeding corrected their hyperphagia, suggesting an acute postprandial regulation of feeding by LCN2. In full agreement with these observations, elevated levels of LCN2 were found in normal-weight women following a high-fat meal, whereas this regulation was lost in obese women (Paton et al. 2013).

Following the increase of its circulating levels, LCN2 crosses the blood-brain barrier and accumulates mainly in the hypothalamus. Supporting a central function, direct delivery of LCN2 in the hypothalamus, reduces food intake and body weight gain as efficiently as its peripheral administration. In the hypothalamus, LCN2 activates neurons in the paraventricular nucleus of the hypothalamus (PVN) as shown by electrophysiological recordings and Fos induction. The PVN is involved in feeding regulation partly as the result of actions of the well-established MC4R anorexigenic pathway. Mice and humans with MC4R defects show hyperphagia and early-onset obesity, increased fat mass, decreased energy expenditure, and hyperinsulinemia (Tao 2010). A series of molecular and biochemical studies showed that LCN2 activates the MC4R pathway, cAMP production, and induction of MC4R target genes, through direct binding to MC4R with an affinity similar to that of its known ligand, $\alpha$ melanocyte-stimulating hormone ( $\alpha \mathrm{MSH})$. Accordingly, LCN2 ability to suppress appetite or accumulate and activate the PVN neurons of the hypothalamus is blunted in $M c 4 r$-depleted mice (Fig. 5). These observations establish a central role for MC4R in mediating the LCN2 anorexigenic response.

The LCN2/MC4R interaction extends to humans as some obese people carrying mutations in MC4R had elevated LCN2 plasma levels as compared with weight-matched people without mutations. This observation is implicit of increased ligand levels in the case of desensitization or inactivation of its cognate GPCR in an attempt to overcome resistance and reestablish homeostasis. Further supporting a compensatory homeostatic response of LCN2, its circulating levels increase in obesity and insulin resistance associated with hyperglycemia in humans, decreased in long-term diabetics, and are inversely correlated with body weight and glycated hemoglobin in diabetic patients (Wang et al. 2007; Yan et al. 2007; Zhang et al. 2008; Alkharfy et al. 2012; De la Chesnaye et al. 2015; Mosialou et al. 2017). Up-regulation of LCN2 in diabetics has also been reported but may be reflective of associated comorbidities such as kidney and cardiovascular disease (Paragas et al. 2012). Given that LCN2 is up-regulated by proinflammatory cytokines to resolve inflammation in adipocytes and macrophages (Zhang et al. 2008; Guo et al. 2014), its up-regulation during obesity may be a protective mechanism against inflammation-induced insulin resistance at the early stages of the disease. Indeed, exogenous LCN2 suppresses appetite and improves glucose 


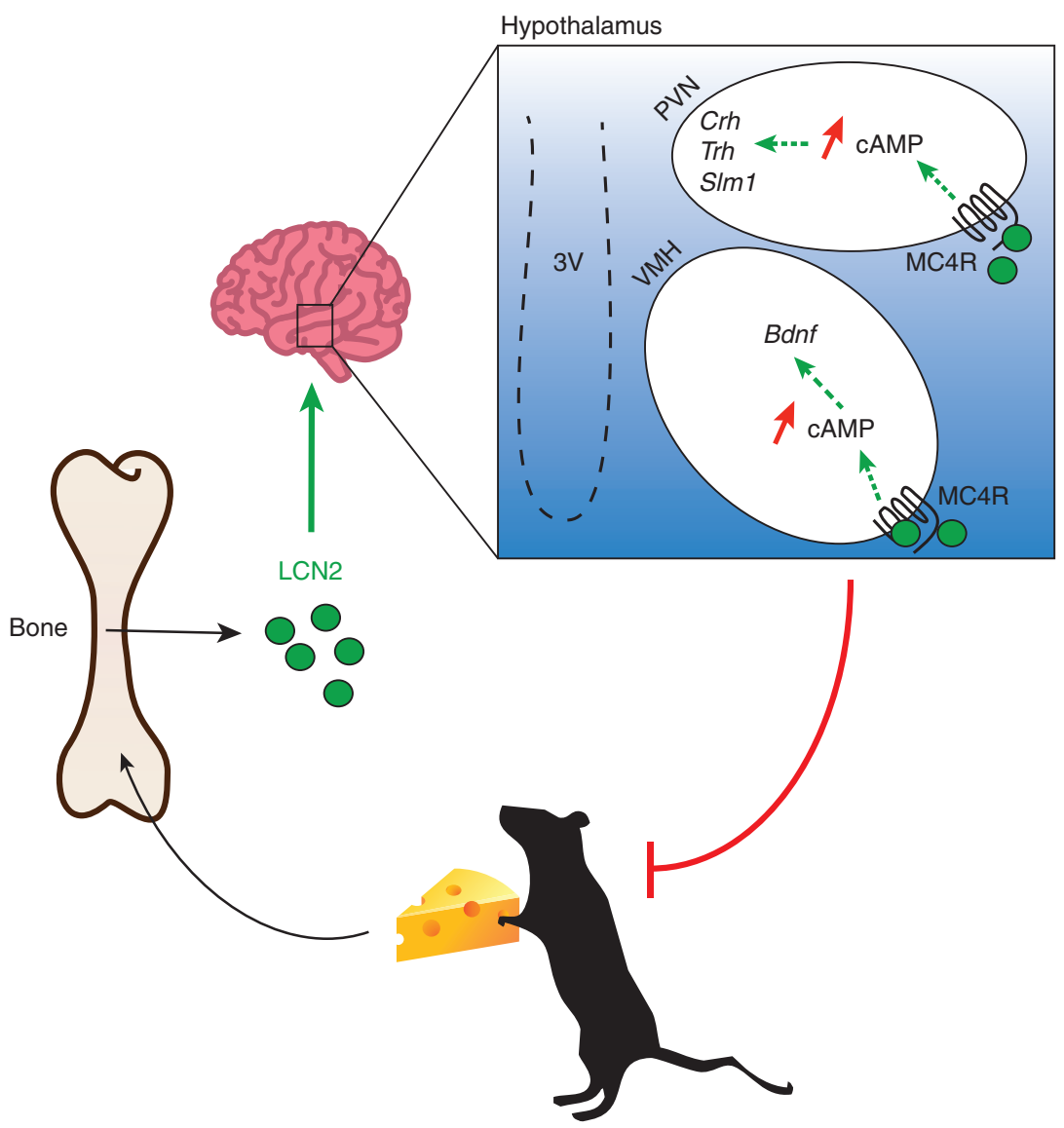

Figure 5. Regulation of food intake by lipocalin-2 (LCN2). Following feeding, LCN2 is released by osteoblasts and accumulates in the hypothalamus where it binds the melanocortin 4 receptor (MC4R) in the paraventricular nucleus of the hypothalamus (PVN) and ventromedial neurons of the hypothalamus (VMH). Activation of MC4R by LCN2 induces cAMP and the expression of Crh, Trh, Sim1, and Bdnf so as to suppress food intake.

metabolism and energy expenditure in $d b / d b$ mice (Mosialou et al. 2017).

Elevated levels of LCN2 have been reported in different pathological settings, mainly associated with the underlying inflammation (Rodvold et al. 2012; Ferreira et al. 2015). Interestingly, $\mathrm{Mc4r}$ expression also increases during inflammation and counteracting its function by MC4R antagonism improves food intake in both acute and chronic inflammatory diseases (Huang et al. 1999; Weyermann et al. 2009; Borges et al. 2011). The up-regulation of LCN2 during inflammation may thus also contribute to disease-associated anorexia.

The regulation of appetite by bone also provides a feedback mechanism to the well-estab- lished central control of bone mass and therefore further illustrates how important the cross talk is between bone and the brain. Similarly, the increase in LCN2 in response to mechanical unloading, countered by physical exercise, or in patients at bed rest may be a homeostatic response to match feeding to reduced caloric needs during periods of inactivity or sedentary behavior (Rucci et al. 2015).

\section{ARE THERE ADDITIONAL BONE-DERIVED HORMONES?}

The identification of the anorexigenic function of LCN2 broadens the spectrum of bone metabolic functions. However, besides food intake, 
P. Mera et al.

OCN-independent effects on fat mass and energy expenditure were observed in mice with partial osteoblast ablation. Gonadal fat was decreased and energy expenditure increased, which was opposite to what would be expected in OCN deficiency. This observation was confirmed by a subsequent study in mice with a decrease in bone mass by $\beta$-catenin deletion in early lineage osteoblasts that led to an OCN-independent increase in food intake and energy expenditure and decreased fat mass (Yao et al. 2017). Neither the decrease in LCN2, which based on previous findings is expected to increase fat mass in its absence, can explain these effects suggesting that an additional factor/s should exist that counteracts the effects of OCN and LCN2 on adiposity.

The regulation of body adiposity by the skeleton may also be fulfilled by osteocyte-derived signals. Partial osteocyte deletion in mice led to severe fat and body weight loss through a yet-tobe-identified cooperation with hypothalamic nuclei regulating energy balance (Sato et al. 2013). Similarly, constitutive and inducible deletion of $G s \alpha$ in osteocytes with concomitant increase in sclerostin levels, an osteocyte-secreted factor, led to the expected decrease in bone mass, but also to a significant decrease in body adiposity caused by induction of beige adipogenesis by sclerostin (Fulzele et al. 2017). In another study, it was reported that $L r p 5$ deletion in osteoblasts and osteocytes, a strong regulator of bone remodeling, resulted in age-related increase in body fat mass and circulating lipid levels with a concomitant reduction in energy expenditure that was attributed to reduced FA $\beta$-oxidation and utilization by bone (Frey et al. 2015). Glucose metabolism does not appear to be altered by genetic manipulations in osteocytes suggesting that osteocyte as compared with osteoblast functions may be limited to the regulation of fat metabolism.

\section{CONCLUDING REMARKS}

Like many other tissues, bone has recently emerged as an unexpected endocrine organ. Bone cells secrete at least two hormones, OCN and LCN2, which are involved in the regulation of energy metabolism. A more complete understanding of the roles, the mechanisms of action and the regulation of these two bone-derived hormones must be achieved before OCN- or LCN2-based therapies for glucose metabolism disorders can be developed for humans. Nonetheless, wherever the future research on bone endocrine function will take us, it is clear that our view of bone has already been irremediably transformed.

\section{ACKNOWLEDGMENTS}

This work is supported by funding from the Canada Research Chair program (M.F.), by the Canadian Institutes of Health Research (M.F., MOP-133652), and by the Natural Sciences and Engineering Research Council of Canada (M.F.).

\section{REFERENCES}

* Reference is also in this collection.

Alkharfy KM, Al-Daghri NM, Vanhoutte PM, Krishnaswamy S, Xu A. 2012. Serum retinol-binding protein 4 as a marker for cardiovascular disease in women. PLoS ONE 7: e48612.

Bonneau J, Ferland G, Karelis AD, Doucet E, Faraj M, Rabasa-Lhoret R, Ferron M. 2017. Association between osteocalcin $\gamma$-carboxylation and insulin resistance in overweight and obese postmenopausal women. J Diabetes Complications 31: 1027-1034.

Borges BC, Rorato R, Avraham Y, da Silva LE, Castro M, Vorobiav L, Berry E, Antunes-Rodrigues J, Elias LL. 2011. Leptin resistance and desensitization of hypophagia during prolonged inflammatory challenge. Am J Physiol Endocrinol Metab 300: E858-E869.

Cornish J, Callon KE, Bava U, Lin C, Naot D, Hill BL, Grey AB, Broom N, Myers DE, Nicholson GC, et al. 2002. Leptin directly regulates bone cell function in vitro and reduces bone fragility in vivo. J Endocrinol 175: 405-415.

Das SK, Sharma NK, Elbein SC. 2010. Analysis of osteocalcin as a candidate gene for type 2 diabetes (T2D) and intermediate traits in Caucasians and African Americans. Dis Markers 28: 281-286.

De la Chesnaye E, Manuel-Apolinar L, Zarate A, Damasio L, Espino N, Revilla-Monsalve MC, Islas-Andrade S. 2015. Lipocalin-2 plasmatic levels are reduced in patients with long-term type 2 diabetes mellitus. Int J Clin Exp Med 8: 2853-2859.

De Toni L, De Filippis V, Tescari S, Ferigo M, Ferlin A, Scattolini V, Avogaro A, Vettor R, Foresta C. 2014. Uncarboxylated osteocalcin stimulates 25 -hydroxy vitamin D production in Leydig cell line through a GPRC6a-dependent pathway. Endocrinology 155: 4266-4274. 
De Toni L, Di Nisio A, Speltra E, Rocca MS, Ghezzi M Zuccarello D, Turiaco N, Ferlin A, Foresta C. 2016a. Polymorphism rs2274911 of GPRC6A as a novel risk factor for testis failure. J Clin Endocrinol Metab 101: 953-961.

De Toni L, Guidolin D, De Filippis V, Tescari S, Strapazzon G, Santa Rocca M, Ferlin A, Plebani M, Foresta C. 2016b. Osteocalcin and sex hormone binding globulin compete on a specific binding site of GPRC6A. Endocrinology 157: 4473-4486.

Di Nisio A, Rocca MS, Fadini GP, De Toni L, Marcuzzo G, Marescotti MC, Sanna M, Plebani M, Vettor R, Avogaro A, et al. 2017. The rs2274911 polymorphism in GPRC6A gene is associated with insulin resistance in normal weight and obese subjects. Clin Endocrinol (Oxf) 86: 185-191.

Ducy P, Amling M, Takeda S, Priemel M, Schilling AF, Beil FT, Shen J, Vinson C, Rueger JM, Karsenty G. 2000. Leptin inhibits bone formation through a hypothalamic relay: A central control of bone mass. Cell 100: 197-207.

Febbraio MA, Pedersen BK. 2002. Muscle-derived interleukin-6: Mechanisms for activation and possible biological roles. FASEB J 16: 1335-1347.

Febbraio MA, Hiscock N, Sacchetti M, Fischer CP, Pedersen BK. 2004. Interleukin-6 is a novel factor mediating glucose homeostasis during skeletal muscle contraction. Diabetes 53: 1643-1648.

Ferreira AC, Da Mesquita S, Sousa JC, Correia-Neves M, Sousa N, Palha JA, Marques F. 2015. From the periphery to the brain: Lipocalin-2, a friend or foe? Prog Neurobiol 131: $120-136$.

Ferron M, Hinoi E, Karsenty G, Ducy P. 2008. Osteocalcin differentially regulates $\beta$ cell and adipocyte gene expression and affects the development of metabolic diseases in wild-type mice. Proc Natl Acad Sci 105: 5266-5270.

Ferron M, Wei J, Yoshizawa T, Del Fattore A, DePinho RA, Teti A, Ducy P, Karsenty G. 2010. Insulin signaling in osteoblasts integrates bone remodeling and energy metabolism. Cell 142: 296-308.

Ferron M, McKee MD, Levine RL, Ducy P, Karsenty G. 2012. Intermittent injections of osteocalcin improve glucose metabolism and prevent type 2 diabetes in mice. Bone 50: $568-575$.

Ferron M, Lacombe J, Germain A, Oury F, Karsenty G. 2015. GGCX and VKORC1 inhibit osteocalcin endocrine functions. J Cell Biol 208: 761-776.

* Frenette P. 2017. Neural regulation of bone marrow. Cold Spring Harb Perspect Med doi: 10.1101/cshperspect. a031344

Frey JL, Li Z, Ellis JM, Zhang Q, Farber CR, Aja S, Wolfgang MJ, Clemens TL, Riddle RC. 2015. Wnt-Lrp5 signaling regulates fatty acid metabolism in the osteoblast. Mol Cell Biol 35: 1979-1991.

Fulzele K, Riddle RC, DiGirolamo DJ, Cao X, Wan C, Chen D, Faugere MC, Aja S, Hussain MA, Bruning JC, et al. 2010. Insulin receptor signaling in osteoblasts regulates postnatal bone acquisition and body composition. Cell 142: 309-319.

Fulzele K, Lai F, Dedic C, Saini V, Uda Y, Shi C, Tuck P, Aronson JL, Liu X, Spatz JM, et al. 2017. Osteocyte-secreted Wnt signaling inhibitor sclerostin contributes to beige adipogenesis in peripheral fat depots. J Bone Miner Res 32: 373-384.
* Galán-Díez M, Cuesta-Domínguez A, Kousteni S. 2017. The bone marrow microenvironment in health and myeloid malignancy. Cold Spring Harb Perspect Med doi: 10.1101/ cshperspect.a031328.

Gao J, Bai T, Ren L, Ding Y, Zhong X, Wang H, Guo Y, Li J, Liu Y, Zhang Y. 2016a. The PLC/PKC/Ras/MEK/Kv channel pathway is involved in uncarboxylated osteocalcin-regulated insulin secretion in rats. Peptides 86: 72-79.

Gao J, Zhong X, Ding Y, Bai T, Wang H, Wu H, Liu Y, Yang J, Zhang Y. 2016b. Inhibition of voltage-gated potassium channels mediates uncarboxylated osteocalcin-regulated insulin secretion in rat pancreatic $\beta$ cells. Eur J Pharmacol 777: 41-48.

Guo H, Jin D, Chen X. 2014. Lipocalin 2 is a regulator of macrophage polarization and NF- $\mathrm{\kappa B} / \mathrm{STAT} 3$ pathway activation. Mol Endocrinol 28: 1616-1628.

Guo H, Jin D, Zhang Y, Wright W, Bazuine M, Brockman DA, Bernlohr DA, Chen X. 2010. Lipocalin-2 deficiency impairs thermogenesis and potentiates diet-induced insulin resistance in mice. Diabetes 59: 1376-1385.

Guo H, Zhang Y, Brockman DA, Hahn W, Bernlohr DA, Chen X. 2012. Lipocalin 2 deficiency alters estradiol production and estrogen receptor signaling in female mice. Endocrinology 153: 1183-1193.

Guo H, Bazuine M, Jin D, Huang MM, Cushman SW, Chen X. 2013. Evidence for the regulatory role of lipocalin 2 in high-fat diet-induced adipose tissue remodeling in male mice. Endocrinology 154: 3525-3538.

Gupte AA, Sabek OM, Fraga D, Minze LJ, Nishimoto SK, Liu JZ, Afshar S, Gaber L, Lyon CJ, Osama Gaber A, et al. 2014. Osteocalcin protects against non-alcoholic steatohepatitis in a mouse model of metabolic syndrome. Endocrinology 155: 4697-4705.

Hill HS, Grams J, Walton RG, Liu J, Moellering DR, Garvey WT. 2014. Carboxylated and uncarboxylated forms of osteocalcin directly modulate the glucose transport system and inflammation in adipocytes. Horm Metab Res 46: 341-347.

Hinoi E, Gao N, Jung DY, Yadav V, Yoshizawa T, Myers MG Jr, Chua SC Jr, Kim JK, Kaestner KH, Karsenty G. 2008. The sympathetic tone mediates leptin's inhibition of insulin secretion by modulating osteocalcin bioactivity. J Cell Biol 183: 1235-1242.

Horsnell H, Baldock PA. 2016. Osteoblastic actions of the neuropeptide Y system to regulate bone and energy homeostasis. Curr Osteoporos Rep 14: 26-31.

Huang QH, Hruby VJ, Tatro JB. 1999. Role of central melanocortins in endotoxin-induced anorexia. Am J Physiol 276: R864-R871.

Huang L, Yang L, Luo L, Wu P, Yan S. 2017. Osteocalcin improves metabolic profiles, body composition and arterial stiffening in an induced diabetic rat model. Exp Clin Endocrinol Diabetes 125: 234-240.

Jun LS, Siddall CP, Rosen ED. 2011. A minor role for lipocalin 2 in high-fat diet-induced glucose intolerance. Am J Physiol Endocrinol Metab 301: E825-E835.

Kajimura D, Lee HW, Riley KJ, Arteaga-Solis E, Ferron M, Zhou B, Clarke CJ, Hannun YA, DePinho RA, Guo EX, et al. 2013. Adiponectin regulates bone mass via opposite central and peripheral mechanisms through FoxO1. Cell Metab 17: 901-915. 
P. Mera et al.

Keller C, Steensberg A, Pilegaard H, Osada T, Saltin B, Pedersen BK, Neufer PD. 2001. Transcriptional activation of the IL-6 gene in human contracting skeletal muscle: Influence of muscle glycogen content. FASEB J 15: 27482750.

* Khosla S, Monroe DG. 2017. Regulation of bone metabolism by sex steroids. Cold Spring Harb Perspect Med doi: 10.1101/cshperspect.a031211.

Kim JG, Sun BH, Dietrich MO, Koch M, Yao GQ, Diano S, Insogna K, Horvath TL. 2015. AgRP neurons regulate bone mass. Cell Rep 13: 8-14.

Korostishevsky M, Malkin I, Trofimov S, Pei Y, Deng HW, Livshits G. 2012. Significant association between body composition phenotypes and the osteocalcin genomic region in normative human population. Bone 51: 688-694.

Kover K, Yan Y, Tong PY, Watkins D, Li X, Tasch J, Hager M Clements M, Moore WV. 2015. Osteocalcin protects pancreatic $\beta$ cell function and survival under high glucose conditions. Biochem Biophys Res Commun 462: 21-26.

* Kronenberg HM. 2017. Regulation of bone remodeling by parathyroid hormone. Cold Spring Harb Perspect Med doi: 10.1101/cshperspect.a031237.

Kunutsor SK, Apekey TA, Laukkanen JA. 2015. Association of serum total osteocalcin with type 2 diabetes and intermediate metabolic phenotypes: Systematic review and meta-analysis of observational evidence. Eur J Epidemiol 30: 599-614.

Lacombe J, Karsenty G, Ferron M. 2013. In vivo analysis of the contribution of bone resorption to the control of glucose metabolism in mice. Mol Metab 2: 498-504.

Law IK, Xu A, Lam KS, Berger T, Mak TW, Vanhoutte PM, Liu JT, Sweeney G, Zhou M, Yang B, et al. 2010. Lipocalin2 deficiency attenuates insulin resistance associated with aging and obesity. Diabetes 59: 872-882.

Lee NK, Sowa H, Hinoi E, Ferron M, Ahn JD, Confavreux C, Dacquin R, Mee PJ, McKee MD, Jung DY, et al. 2007. Endocrine regulation of energy metabolism by the skeleton. Cell 130: 456-469.

Legroux-Gerot I, Vignau J, Collier F, Cortet B. 2005. Bone loss associated with anorexia nervosa. Joint Bone Spine 72: 489-495.

Liu C, Wo J, Zhao Q, Wang Y, Wang B, Zhao W. 2015. Association between serum total osteocalcin level and type 2 diabetes mellitus: A systematic review and metaanalysis. Horm Metab Res 47: 813-819.

Mera P, Laue K, Ferron M, Confavreux C, Wei J, Galan-Diez M, Lacampagne A, Mitchell SJ, Mattison JA, Chen Y, et al. 2016a. Osteocalcin signaling in myofibers is necessary and sufficient for optimum adaptation to exercise. Cell Metab 23: 1078-1092.

Mera P, Laue K, Wei J, Berger JM, Karsenty G. 2016b. Osteocalcin is necessary and sufficient to maintain muscle mass in older mice. Mol Metab 5: 1042-1047.

Misra M, Klibanski A. 2011. The neuroendocrine basis of anorexia nervosa and its impact on bone metabolism. Neuroendocrinology 93: 65-73.

Mosialou I, Shikhel S, Liu JM, Maurizi A, Luo N, He Z, Huang Y, Zong H, Friedman RA, Barasch J, et al. 2017. MC4R-dependent suppression of appetite by bone-derived lipocalin 2. Nature 543: 385-390.
* Murshed M. 2017. Bone mineralization. Cold Spring Harb Perspect Med doi: 10.1101/cshperspect.a031229.

Nielsen AR, Mounier R, Plomgaard P, Mortensen OH, Penkowa M, Speerschneider T, Pilegaard H, Pedersen BK. 2007. Expression of interleukin-15 in human skeletal muscle effect of exercise and muscle fibre type composition. J Physiol 584: 305-312.

Olson EN, Arnold HH, Rigby PW, Wold BJ. 1996. Know your neighbors: Three phenotypes in null mutants of the myogenic bHLH gene MRF4. Cell 85: 1-4.

Oury F, Sumara G, Sumara O, Ferron M, Chang H, Smith CE, Hermo L, Suarez S, Roth BL, Ducy P, et al. 2011. Endocrine regulation of male fertility by the skeleton. Cell 144: 796-809.

Oury F, Ferron M, Huizhen W, Confavreux C, Xu L, Lacombe J, Srinivas P, Chamouni A, Lugani F, Lejeune H, et al. 2013a. Osteocalcin regulates murine and human fertility through a pancreas-bone-testis axis. J Clin Invest 123: 2421-2433.

Oury F, Khrimian L, Denny CA, Gardin A, Chamouni A, Goeden N, Huang YY, Lee H, Srinivas P, Gao XB, et al. 2013b. Maternal and offspring pools of osteocalcin influence brain development and functions. Cell 155: 228-241.

Paragas N, Qiu A, Hollmen M, Nickolas TL, Devarajan P, Barasch J. 2012. NGAL-Siderocalin in kidney disease. Biochim Biophys Acta 1823: 1451-1458.

Paton CM, Rogowski MP, Kozimor AL, Stevenson JL, Chang H, Cooper JA. 2013. Lipocalin-2 increases fat oxidation in vitro and is correlated with energy expenditure in normal weight but not obese women. Obesity 21: E640-E648.

Pedersen BK, Febbraio MA. 2008. Muscle as an endocrine organ: Focus on muscle-derived interleukin-6. Physiol Rev 88: 1379-1406.

Pi M, Quarles LD. 2012. Multiligand specificity and wide tissue expression of GPRC6A reveals new endocrine networks. Endocrinology 153: 2062-2069.

Pi M, Chen L, Huang MZ, Zhu W, Ringhofer B, Luo J, Christenson L, Li B, Zhang J, Jackson PD, et al. 2008. GPRC6A null mice exhibit osteopenia, feminization and metabolic syndrome. PLoS ONE 3: e3858.

Pi M, Kapoor K, Ye R, Kenneth Nishimoto S, Smith JC, Baudry J, Darryl Quarles L. 2016. Evidence for osteocalcin binding and activation of GPRC6A in $\beta$-cells. Endocrinology 157: 1866-1880.

Rached MT, Kode A, Silva BC, Jung DY, Gray S, Ong H, Paik JH, DePinho RA, Kim JK, Karsenty G, et al. 2010. FoxO1 expression in osteoblasts regulates glucose homeostasis through regulation of osteocalcin in mice. J Clin Invest 120: $357-368$.

Rodvold JJ, Mahadevan NR, Zanetti M. 2012. Lipocalin 2 in cancer: When good immunity goes bad. Cancer Lett 316: 132-138.

* Roodman DGDG. 2017. Multiple myeloma and bone. Cold Spring Harb Perspect Med doi: 10.1101/cshperspect. a031286.

Rucci N, Capulli M, Piperni SG, Cappariello A, Lau P, Frings-Meuthen P, Heer M, Teti A. 2015. Lipocalin 2: A new mechanoresponding gene regulating bone homeostasis. J Bone Miner Res 30: 357-368. 
Sabek OM, Nishimoto SK, Fraga D, Tejpal N, Ricordi C, Gaber AO. 2015. Osteocalcin effect on human $\beta$-cells mass and function. Endocrinology 156: 3137-3146.

Sato M, Asada N, Kawano Y, Wakahashi K, Minagawa K, Kawano H, Sada A, Ikeda K, Matsui T, Katayama Y. 2013. Osteocytes regulate primary lymphoid organs and fat metabolism. Cell Metab 18: 749-758.

Steenbergen RD, OudeEngberink VE, Kramer D, Schrijnemakers HF, Verheijen RH, Meijer CJ, Snijders PJ. 2002. Down-regulation of GATA-3 expression during human papillomavirus-mediated immortalization and cervical carcinogenesis. Am J Pathol 160: 1945-1951.

Steensberg A, van Hall G, Osada T, Sacchetti M, Saltin B, Klarlund Pedersen B. 2000. Production of interleukin-6 in contracting human skeletal muscles can account for the exercise-induced increase in plasma interleukin-6. J Physiol 529: 237-242.

Tamura T, Udagawa N, Takahashi N, Miyaura C, Tanaka S, Yamada Y, Koishihara Y, Ohsugi Y, Kumaki K, Taga T, et al. 1993. Soluble interleukin-6 receptor triggers osteoclast formation by interleukin 6. Proc Natl Acad Sci 90: 11924-11928.

Tao YX. 2010. The melanocortin-4 receptor: Physiology, pharmacology, and pathophysiology. Endocrine Rev 31: 506-543.

Teitelbaum SL, Ross FP. 2003. Genetic regulation of osteoclast development and function. Nat Rev Genet 4: 638649.

van Hall G, Steensberg A, Sacchetti M, Fischer C, Keller C, Schjerling P, Hiscock N, Moller K, Saltin B, Febbraio MA et al. 2003. Interleukin-6 stimulates lipolysis and fat oxidation in humans. J Clin Endocrinol Metab 88: 30053010.

Wang Y, Lam KS, Kraegen EW, Sweeney G, Zhang J, Tso AW, Chow WS, Wat NM, Xu JY, Hoo RL, et al. 2007. Lipocalin-2 is an inflammatory marker closely associated with obesity, insulin resistance, and hyperglycemia in humans. Clin Chem 53: 34-41.

Wei J, Hanna T, Suda N, Karsenty G, Ducy P. 2014a. Osteocalcin promotes $\beta$-cell proliferation during development and adulthood through Gprc6a. Diabetes 63: 1021-1031.

Wei W, Motoike T, Krzeszinski JY, Jin Z, Xie XJ, Dechow PC Yanagisawa M, Wan Y. 2014b. Orexin regulates bone re- modeling via a dominant positive central action and a subordinate negative peripheral action. Cell Metab 19: 927-940.

Weyermann P, Dallmann R, Magyar J, Anklin C, Hufschmid M, Dubach-Powell J, Courdier-Fruh I, Hennebohle M, Nordhoff S, Mondadori C. 2009. Orally available selective melanocortin-4 receptor antagonists stimulate food intake and reduce cancer-induced cachexia in mice. PLoS ONE 4: e4774.

* Whyte MP. 2017. Hypophosphatasia. Cold Spring Harb Perspect Med doi: 10.1101/cshperspect.a031567.

Yamauchi T, Kamon J, Waki H, Terauchi Y, Kubota N, Hara K, Mori Y, Ide T, Murakami K, Tsuboyama-Kasaoka N, et al. 2001. The fat-derived hormone adiponectin reverses insulin resistance associated with both lipoatrophy and obesity. Nat Med 7: 941-946.

Yan QW, Yang Q, Mody N, Graham TE, Hsu CH, Xu Z, Houstis NE, Kahn BB, Rosen ED. 2007. The adipokine lipocalin 2 is regulated by obesity and promotes insulin resistance. Diabetes 56: 2533-2540.

Yao Q, Yu C, Zhang X, Zhang K, Guo J, Song L. 2017. Wnt/ $\beta$-catenin signaling in osteoblasts regulates global energy metabolism. Bone 97: 175-183.

Yoshikawa Y, Kode A, Xu L, Mosialou I, Silva BC, Ferron M, Clemens TL, Economides AN, Kousteni S. 2011. Genetic evidence points to an osteocalcin-independent influence of osteoblasts on energy metabolism. J Bone Miner Res 26: 2012-2025.

Zhang J, Wu Y, Zhang Y, Leroith D, Bernlohr DA, Chen X. 2008. The role of lipocalin 2 in the regulation of inflammation in adipocytes and macrophages. Mol Endocrinol 22: 1416-1426.

Zhang Y, Guo H, Deis JA, Mashek MG, Zhao M, Ariyakumar D, Armien AG, Bernlohr DA, Mashek DG, Chen X. 2014. Lipocalin 2 regulates brown fat activation via a nonadrenergic activation mechanism. J Biol Chem 289: 22063-22077.

Zhou B, Li H, Liu J, Xu L, Zang W, Wu S, Sun H. 2013. Intermittent injections of osteocalcin reverse autophagic dysfunction and endoplasmic reticulum stress resulting from diet-induced obesity in the vascular tissue via the NFкB-p65-dependent mechanism. Cell Cycle 12: $1901-$ 1913. 


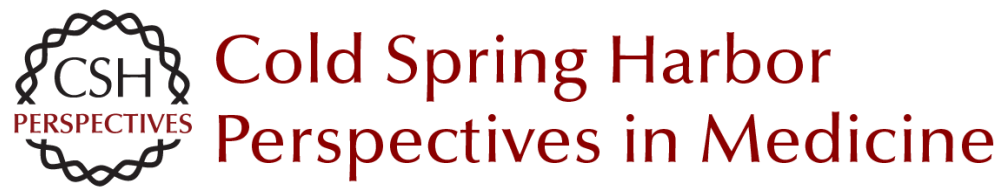

\section{Regulation of Energy Metabolism by Bone-Derived Hormones}

Paula Mera, Mathieu Ferron and loanna Mosialou

Cold Spring Harb Perspect Med 2018; doi: 10.1101/cshperspect.a031666 originally published online August 4, 2017

\section{Subject Collection Bone: A Regulator of Physiology}

\section{Mechanism of Bone Mineralization} Monzur Murshed

Neural Regulation of Bone and Bone Marrow Maria Maryanovich, Shoichiro Takeishi and Paul S. Frenette

Regulation of Bone Remodeling by Parathyroid Hormone Marc N. Wein and Henry M. Kronenberg

The Bone Marrow Microenvironment in Health and Myeloid Malignancy

Marta Galán-Díez, Álvaro Cuesta-Domínguez and Stavroula Kousteni

The Biology of Bone Metastasis

Mark Esposito, Theresa Guise and Yibin Kang

\section{Bone Remodeling and the Microbiome} Roberto Pacifici
Osteoimmunology
Kazuo Okamoto and Hiroshi Takayanagi

Multiple Myeloma and Bone: The Fatal Interaction Silvia Marino and G. David Roodman

Biology of Bone: The Vasculature of the Skeletal System

Emma C. Watson and Ralf H. Adams

Regulation of Energy Metabolism by

Bone-Derived Hormones

Paula Mera, Mathieu Ferron and Ioanna Mosialou

\section{Biology of Fibroblast Growth Factor 23: From \\ Physiology to Pathology \\ Marie Courbebaisse and Beate Lanske}

Regulation of Bone Metabolism by Sex Steroids Sundeep Khosla and David G. Monroe

For additional articles in this collection, see http://perspectivesinmedicine.cshlp.org/cgi/collection/ 